\title{
OSCILLATION THEORY FOR A CLASS OF SECOND ORDER QUASILINEAR DIFFERENCE EQUATIONंS
}

\author{
E. THANDAPANI AND R. ARUL
}

\begin{abstract}
In this paper the authors establish necessary and sufficient conditons for the second order quasilinear difference equation

$$
\triangle\left(a_{n}\left(\Delta y_{n}\right)^{\alpha *}\right)+f\left(n, y_{n+1}\right)=0, \quad n \in N\left(n_{0}\right)
$$

to have various types of nonoscillatory solutions. In addition, in the case when (E) is either strongly superlinear or strongly sublinear, they establish necessary and sufficient conditions for all solutions to oscillate.
\end{abstract}

\section{Introduction}

Determining oscillation criteria for difference equations has attracted a great deal of attention in the last several years see, for example [1, 6, 7, 9-11, 13-16] and the references contained therein. In this paper we study the oscillatory and nonoscillatory behavior of solutions of quasilinear difference equations of the type

$$
\triangle\left(a_{n}\left(\triangle y_{n}\right)^{\alpha_{*}}+f\left(n, y_{n+1}\right)=0, \quad n \in N\left(n_{0}\right)\right.
$$

where $\left(\triangle y_{n}\right)^{\alpha_{*}}=\left|\Delta y_{n}\right|^{\alpha} \operatorname{sgn} \triangle y_{n}, N\left(n_{0}\right)=\left\{n_{0}, n_{0}+1, \ldots\right\}, n_{0} \in \mathbb{N}=\{0,1,2, \ldots\}, \triangle$ is the forward difference operator defined by $\triangle y_{n}=y_{n+1}-y_{n},\left\{a_{n}\right\}$ is a real sequence with $a_{n}>0$ for all $n \in N\left(n_{0}\right), \sum_{n=n_{0}}^{\infty} a_{n}^{-1 / \alpha}<\infty, \alpha>0$ is a constant and $f: N\left(n_{0}\right) \times R \rightarrow R$. is continuous, $u f(n, u)>0$ for $u \neq 0$ and $f(n, u)$ is nondecreasing in $u$ for each fixed $n \in N\left(n_{0}\right)$.

By a solution of equation (1) we mean a nontrivial sequence $\left\{y_{n}\right\}$ satisfying (1) for all $n \in N\left(n_{0}\right)$. A solution $\left\{y_{n}\right\}$ is said to be oscillatory if it is neither eventually positive nor eventually negative, and nonoscillatory otherwise.

First, a classification into three classes of nonoscillatory solutions of equation (1) is given according to their asymptotic behavior as $n \rightarrow \infty$, and conditions are obtained for each of the three classes to be nonempty. Next, Criteria are presented for characterising the oscillation of all solutions of equation (1) with the strongly superlinear or strongly

Received November 23, 1996.

1991 Mathematics Subject Classification. 39A10.

Key words and phrases. Nonoscillatory solutions, oscillation, quasilinear difference equations. 
sublinear condition. The results, in conjunction with the results obtained in [12] for equation (1) with $\left\{a_{n}\right\}$ satisfying $\sum_{n=n_{0}}^{\infty} a_{n}^{-1 / \alpha}=\infty$, provide a natural generalization of the oscillation theory so far developed for the equation

$$
\triangle\left(a_{n} \triangle y_{n}\right)+f\left(n, y_{n+1}\right)=0
$$

beginning with Hooker and Patula [3], Kulenovic and Budincevic [5], He [4], Szmanda [8] and Zhang [17]. Examples which dwell upon the importance of our results are also included.

\section{Nonoscillation Theorems}

We begin by classifying all possible nonoscillatory solutions of equation (1) according to their asymptotic behavior at infinity. This classification is based on the following Lemma.

Lemma 1. If $\left\{y_{n}\right\}$ is a nonoscillatory solution of equation (1), then there exists positive constants $c_{1}, c_{2}$ and $n_{1} \in N\left(n_{0}\right)$ such that

$$
c_{1} \eta_{\alpha}(n) \leq\left|y_{n}\right| \leq c_{2} \quad \text { for } \quad n \in N\left(n_{1}\right)
$$

where

$$
\eta_{\alpha}(n)=\sum_{s=n}^{\infty} a_{n}^{-1 / \alpha}
$$

Proof. Let $\left\{y_{n}\right\}$ be a nonoscillatory solution of equation (1), and without loss of generality, assume that $\left\{y_{n}\right\}$ is eventually positive. Then $\left\{a_{n}\left(\Delta y_{n}\right)^{\alpha_{*}}\right\}$ is eventually decreasing, so $\left\{\Delta y_{n}\right\}$ is eventually of constant sign. If $\triangle y_{n}>0$ for $n \in N\left(n_{1}\right)$ for some $n_{1} \geq n_{0}$, then $a_{n}\left(\triangle y_{n}\right)^{\alpha} \leq a_{n_{1}}\left(\triangle y_{n_{1}}\right)^{\alpha}$ for $n \in N\left(n_{1}\right)$, so

$$
\Delta y_{n} \leq \frac{\left(a_{n_{1}}\right)^{1 / \alpha} \Delta y_{n_{1}}}{\left(a_{n}\right)^{1 / \alpha}} \text { for } n \in N\left(n_{1}\right)
$$

Summing the last inequality from $n_{1}$ to $n-1$, we have

$$
\begin{aligned}
y_{n} & \leq y_{n_{1}}+\left(a_{n_{1}}\right)^{1 / \alpha} \Delta y_{n_{1}} \sum_{s=n_{1}}^{n-1}\left(a_{s}\right)^{-1 / \alpha} \\
& \leq y_{n_{1}}+\left(a_{n_{1}}\right)^{1 / \alpha} \Delta y_{n_{1}} \eta_{\alpha}\left(n_{1}\right), \quad n \in N\left(n_{1}\right) .
\end{aligned}
$$

This proves the right half of the inequality in (2). If $\triangle y_{n}<0$ for $n \in N\left(n_{1}\right)$, then, since $a_{n}\left(\Delta y_{n}\right)^{\alpha_{*}}=-a_{n}\left(-\Delta y_{n}\right)^{\alpha}$, we see that

$$
a_{s}\left(-\triangle y_{s}\right)^{\alpha} \geq a_{n}\left(-\triangle y_{n}\right)^{\alpha}, \quad s \geq n \in N\left(n_{1}\right),
$$


or

$$
-\left(a_{s}\right)^{1 / \alpha} \triangle y_{s} \geq-\left(a_{n}\right)^{1 / \alpha} \triangle y_{n}, \quad s \geq n \in N\left(n_{1}\right) .
$$

Dividing (4) by $\left(a_{s}\right)^{1 / \alpha}$ and summing from $n$ to $j-1$, we have

$$
y_{n}>y_{n}-y_{j} \geq-\left(a_{n}\right)^{1 / \alpha} \Delta y_{n} \sum_{s=n}^{j-1}\left(a_{s}\right)^{-1 / \alpha}, \quad j>n,
$$

which, in the limit as $j \rightarrow \infty$, gives

$$
y_{n} \geq-\left(a_{n}\right)^{1 / \alpha} \triangle y_{n} \eta_{\alpha}(n), \quad n \in N\left(n_{1}\right) .
$$

Combining (5) with the inequality

$$
-\left(a_{n}\right)^{1 / \alpha} \triangle y_{n} \geq-\left(a_{n_{1}}\right)^{1 / \alpha} \triangle y_{n_{1}}, \quad n \in N\left(n_{1}\right)
$$

which is implied by (4), we find

$$
y_{n} \geq-\left(a_{n_{1}}\right)^{1 / \alpha} \triangle y_{n_{1}} \eta_{\alpha}(n), \quad n \in N\left(n_{1}\right) .
$$

This proves the left half of the inequality in (2).

A similar argument holds if $\left\{y_{n}\right\}$ is eventually negative.

Lemma 1 shows that the following three types of asymptotic behavior are possible for nonoscillatory solutions $\left\{y_{n}\right\}$ of equation (1):

$$
\begin{aligned}
& \text { (I) } \lim _{n \rightarrow \infty} y_{n}=\text { constant } \neq 0 ; \\
& \text { (II) } \lim _{n \rightarrow \infty} y_{n}=0 \text { and } \cdot \lim _{n \rightarrow \infty} \frac{\left|y_{n}\right|}{\eta_{\alpha}(n)}=\infty ; \\
& \text { (III) } \lim _{n \rightarrow \infty} \frac{\left|y_{n}\right|}{\eta_{\alpha}(n)}=\text { constant }>0 .
\end{aligned}
$$

First, we characterize the type (I) solutions of equation (1).

Theorem 2. A necessary and sufficient condition for equation (1) to have type (I) nonoscillatory solution $\left\{y_{n}\right\}$ is that

$$
\sum_{n=n_{0}}^{\infty}\left(\frac{1}{a_{n}} \sum_{s=n_{0}}^{n-1}|f(s, c)|\right)^{1 / \alpha}<\infty
$$

for some nonzero constant $c$.

Proof. (Necessity) Let $\left\{y_{n}\right\}$ be a type (I) solution of equation (1) which is positive for $n \in N\left(n_{1}\right)$. Then, there is a constant $c>0$ such that $y_{n}>c$ for $n \in N\left(n_{1}\right)$. If $\Delta y_{n}>0$ for $n \in N\left(n_{1}\right)$, a summation of equation (1) shows that

$$
\sum_{n=n_{1}}^{\infty} f(n, c) \leq \sum_{n=n_{1}}^{\infty} f\left(n, y_{n+1}\right) \leq a_{n_{1}}\left(\triangle y_{n_{1}}\right)^{\alpha}<\infty
$$


from which (6) follows easily. If $\Delta y_{n}<0$ for $n \in N\left(n_{1}\right)$, then summation of (1) gives

$$
\sum_{s=n_{1}}^{n-1} f\left(s, y_{s+1}\right)=a_{n_{1}}\left(\triangle y_{n_{1}}\right)^{\alpha_{*}}-a_{n}\left(\Delta y_{n}\right)^{\alpha_{*}}<a_{n}\left(-\triangle y_{n}\right)^{\alpha}, \quad n \in N\left(n_{1}\right)
$$

or

$$
\left(\frac{1}{a_{n}} \sum_{s=n_{1}}^{n-1} f\left(s, y_{s+1}\right)\right)^{1 / \alpha} \leq-\triangle y_{n}, \quad n \in N\left(n_{1}\right) .
$$

Summing the last inequality from $n_{1}$ to $j$ and using the fact that $c_{1} \leq y_{n} \leq c_{2}, n \in N\left(n_{1}\right)$, for some positive constants $c_{1}$ and $c_{2}$, we see that

$$
\sum_{n=n_{1}}^{j}\left(\frac{1}{a_{n}} \sum_{s=n_{1}}^{n-1} f\left(s, c_{1}\right)\right)^{1 / \alpha} \leq y_{n_{1}}-y_{j+1}<c_{2}
$$

for any $j>n_{1}$, which clearly implies (6).

Sufficiency. Suppose that the constant in (6) is positive and $n_{2} \geq n_{1}$ be large enough so that

$$
\sum_{n=n_{2}}^{\infty}\left(\frac{1}{a_{n}} \sum_{s=n_{2}}^{n-1} f(s, c)\right)^{1 / \alpha} \leq c / 2
$$

Consider the Banach space $B_{n_{2}}$ of all real sequences $y=\left\{y_{n}\right\}, n \in N\left(n_{2}\right)$ with the supremum norm $\|y\|=\sup _{n \in N\left(n_{2}\right)}\left|y_{n}\right|$. We define a subset of $B_{n_{2}}$ as

$$
S=\left\{y \in B_{n_{2}}: \frac{c}{2} \leq y_{n} \leq, n \in N\left(n_{2}\right)\right\} .
$$

Clearly, $S$ is a bounded, closed and convex subset of $B_{n_{2}}$. Now we define an operator $T: S \rightarrow B_{n_{2}}$ as follows

$$
T y_{n}=c-\sum_{s=n_{2}}^{n-1}\left(\frac{1}{a_{s}} \sum_{t=n_{2}}^{s-1} f\left(t, y_{t+1}\right)\right)^{1 / \alpha}, \quad n \in N\left(n_{2}\right) .
$$

From the hypothesis this operator $T$ is continuous, and for $y \in S$, we have

$$
\frac{c}{2} \leq T y_{n} \leq c \quad \text { for } \quad n \in N\left(n_{2}\right) .
$$

Thus, $T S \subseteq S$. Therefore, by the Schauder fixed point theorem $T$ has a fixed point $y \in S$. It is clear that $\delta\left\{y_{n}\right\}$ is a positive solution of equation (1) and $\lim _{n \rightarrow \infty} y_{n}=$ constant belongs to $[c / 2, c]$. This implies that $\left\{y_{n}\right\}$ is a type (I) solution of equation (1). This completes the proof.

Theorem 3. A necessary and sufficient condition for equation (1) to have a type (III) nonoscillatory solution $\left\{y_{n}\right\}$ is that

$$
\sum_{n=n_{0}}^{\infty} \mid f\left(n, c \eta_{\alpha}(n+1) \mid<\infty\right.
$$


for some nonzero constant c.

Proof. (Necessity). Let $\left\{y_{n}\right\}$ be a type (III) solution of equation (1) which is eventually positive. There are constants $c_{1}, c_{2}>0$ and $n_{1} \in N\left(n_{0}\right)$ such that

$$
c_{1} \eta_{\alpha}(n) \leq y_{n} \leq c_{2} \eta_{\alpha}(n) \text { for } n \in N\left(n_{1}\right) .
$$

We may suppose that $\triangle y_{n}<0$ for $n \in N\left(n_{1}\right)$. From equation (1) we have

$$
\sum_{s=n_{1}}^{n-1} f\left(s, y_{s+1}\right) \leq a_{n}\left(-\triangle y_{n}\right)^{\alpha}, \quad n \in N\left(n_{1}\right),
$$

which is obtained from (7). Combining the last inequality with the inequality

$$
a_{n}\left(-\triangle y_{n}\right)^{\alpha} \leq\left(\frac{y_{n}}{\eta_{\alpha}(n)}\right)^{\alpha}, \quad n \in N\left(n_{1}\right)
$$

which is equivalant to (5), we obtain

$$
\sum_{s=n_{1}}^{n-1} f\left(s, c_{1} \eta_{\alpha}(s+1)\right) \leq \sum_{s=n_{1}}^{n-1} f\left(s, y_{s+1}\right) \leq\left(\frac{y_{n}}{\eta_{\alpha}(n)}\right)^{\alpha} \leq c_{2}^{\alpha}, \quad n \in N\left(n_{1}\right)
$$

which gives (8) in the limit as $n \rightarrow \infty$. A similar argument holds if $\left\{y_{n}\right\}$ is an eventually negative solution of type (III) of equation (1).

Sufficiency. We may assume that constant $c$ in (8) is positive. Let $k>0$ be a constant such that $2 k \leq c^{\alpha}$ and take $n_{1} \in N\left(n_{0}\right)$ so that

$$
\sum_{n=n_{1}}^{\infty} f\left(n,(2 k)^{1 / \alpha} \eta_{\alpha}(n+1)\right) \leq k
$$

Let $B_{n_{1}}$ be the same Banach space as in the proof of Theorem 2. Define a subset $S$ and an operator $T: S \rightarrow B_{n_{1}}$, by

$$
\begin{aligned}
& S=\left\{y \in B_{n_{1}}: k^{1 / \alpha} \eta_{\alpha}(n) \leq y_{n} \leq(2 k)^{1 / \alpha} \eta_{\alpha}(n), \quad n \in N\left(n_{1}\right)\right\} \\
& T y_{n}=\sum_{s=n}^{\infty}\left(\frac{1}{a_{s}}\left(k+\sum_{t=n_{1}}^{s-1} f\left(t, y_{t+1}\right)\right)\right)^{1 / \alpha}, \quad n \in N\left(n_{1}\right) .
\end{aligned}
$$

As in the proof of Theorem 2, we can show that $T$ satisfies the assumptions of the Schauder fixed point theorem. Hence, there exists $y \in S$ such that $T y=y$, that is,

$$
y_{n}=\sum_{s=n}^{\infty}\left(\frac{1}{a_{s}}\left(k+\sum_{t=n_{1}}^{s-1} f\left(t, y_{t+1}\right)\right)\right)^{1 / \alpha}, \quad n \in N\left(n_{1}\right) .
$$

From this equation, it follows that $\left\{y_{n}\right\}$ is a positive solution of equation (1), and that, by Stolz's theorem [2],

$$
\lim _{n \rightarrow \infty} \frac{y_{n}}{\eta_{\alpha}(n)}=\text { constant } \in\left[k^{1 / \alpha}(2 k)^{1 / \alpha}\right]
$$


Thus $\left\{y_{n}\right\}$ is a solution of type (III) of equation (1). This completes the proof.

We next give sufficient conditions for the existence of a type (II) solution of equation (1).

Theorem 4. The equation (1) has a nonoscillatory solution of type (II) if

$$
\sum_{n=n_{0}}^{\infty}\left(\frac{1}{a_{n}} \sum_{s=n_{0}}^{n-1}|f(s, c)|\right)^{1 / \alpha}<\infty
$$

for some nonzero constant $c$ and

$$
\sum_{n=n_{0}}^{\infty} \mid f\left(n, d \eta_{\alpha}(n+1) \mid=\infty\right.
$$

for any constant $d \neq 0$ with $c d>0$.

Proof. Suppose that the $c$ in (9) is positive. Choose $k>0$ such that $(2 k)^{1 / \alpha}\left(\eta_{\alpha}\left(n_{0}\right)+\right.$ 1) $\leq c$ and let $n_{1} \in N\left(n_{0}\right)$ be large enough so that

$$
\sum_{n=n_{1}}^{\infty}\left(\frac{1}{a_{n}} \sum_{s=n_{1}}^{n-1} f(s, c)\right)^{1 / \alpha} \leq k^{1 / \alpha} .
$$

Let $B_{n_{1}}$ be the same as in the proof of Theorem 3, and define a subset $S$ and an operator $T: S \rightarrow B_{n_{1}}$ by

$$
\begin{aligned}
& S=\left\{y \in B_{n_{1}}: k^{1 / \alpha} \eta_{\alpha}(n) \leq y_{n} \leq c, \quad n \in N\left(n_{1}\right)\right\} \\
& T y_{n}=\sum_{s=n}^{\infty}\left(\frac{1}{a_{s}}\left(k+\sum_{t=n_{1}}^{s-1} f\left(t, y_{t+1}\right)\right)\right)^{1 / \alpha}, \quad n \in N\left(n_{1}\right) .
\end{aligned}
$$

Similar to the proof of Theorem 2, we can show that the mapping satisfies the assumptions of Schauder fixed point theorem. Hence there exists $y \in S$ such that $T y=y$; that is, $\left\{y_{n}\right\}$ is a nonoscillatory solution of equation (1) satisfying $\lim _{n \rightarrow \infty} y_{n}=0$. To see that $\frac{y_{n}}{\eta_{\alpha}(n)} \rightarrow \infty$ as $n \rightarrow \infty$, we use Stolz's theorem [2] and (10) to obtain

$$
\begin{aligned}
\lim _{n \rightarrow \infty} \frac{y_{n}}{\eta_{\alpha}(n)} & =\lim _{n \rightarrow \infty} \frac{\Delta y_{n}}{\Delta \eta_{\alpha}(n)}=\lim _{n \rightarrow \infty}\left(k+\sum_{s=n_{1}}^{n-1} f\left(s, y_{s+1}\right)\right)^{1 / \alpha} \\
& \geq \lim _{n \rightarrow \infty}\left(k+\sum_{s=n_{1}}^{n-1} f\left(s, k^{1 / \alpha} \eta_{\alpha}(s+1)\right)\right)^{1 / \alpha}=\infty
\end{aligned}
$$

Example 1 . Consider the difference eqution

$$
\triangle\left(2^{\lambda_{n}}\left(\triangle y_{n}\right)^{\alpha_{*}}\right)+2^{\mu n} y_{n+1}^{\beta_{*}}=0, \quad n \in \mathbb{N}
$$


where $y_{n+1}^{\beta *}=\left|y_{n+1}\right|^{\beta} \operatorname{sgn} y_{n+1}$, and $\alpha>0, \beta>0, \lambda>0$ and $\mu$ are constants. Applying Theorem 2 and 3 to this equation, we see that:

(i) Equation (11) possesses a nonoscillatory solution of type (I) if and only if $\mu<\lambda$;

(ii) Equation (11) possesses a nonoscillatory solution of type III (which behaves like a constant multiple of $2^{(-\lambda / \alpha) n}$ as $\left.n \rightarrow \infty\right)$ if and only if $\mu / \lambda<\beta / \alpha$.

Theorem 4 implies that equation (11) possesses a nonoscillatory solution of type (II) if $\beta / \alpha<\mu / \lambda<1$.

\section{Oscillation Theorems}

In this section we study the oscillatory behavior of solutions of equation (1). In view of the results of Hooker ane Patula [3] and Zhang [17], it is reasonable to expect that a characterization of oscillation for equation (1) can be obtained under suitable additional conditions on the nonlinear funciton $f$.

Definition 5. (i) The equation (1) (or the function $f(n, u)$ ) is said to be strongly superlinear if there is a constant $\gamma>\alpha$ such that $|u|^{-\gamma} f(n, u)$ is nondecreasing in $u$ for each fixed $n \in N\left(n_{0}\right)$.

(ii) The equation (1) (or the function $f(n, u)$ ) is said to be strongly sublinear if there is a positive constant $\gamma<\alpha$ such that $|u|^{-\gamma} f(n, u)$ is nonincreasing in $u$ for each fixed $n \in N\left(n_{0}\right)$.

Theorem 6. Let equation (1) be strongly superlinear. All solutions of equation (1) are oscillatory if and only if

$$
\sum_{n=n_{0}}^{\infty} \mid f\left(n, c \eta_{\alpha}(n+1) \mid=\infty\right.
$$

for every nonzero constant $c$.

Proof. The necessary part follows from Theorem 3. To prove sufficiency suppose that equation (1) has a nonoscillatory solution $\left\{y_{n}\right\}$, say $y_{n}>0$ for $n \geq n_{1} \in N\left(n_{0}\right)$. Then, either $\Delta y_{n}>0$ for $n \in N\left(n_{1}\right)$ or $\Delta y_{n}<0$ for $n \in N\left(n_{1}\right)$.

In the first case, it is easy to see that $\sum_{n=n_{1}}^{\infty} f\left(n, c_{1}\right)<\infty$ for some $c_{1}>0$. Since $\eta_{\alpha}(n) \rightarrow 0$ as $n \rightarrow \infty$, there exists an integer $n_{2} \in N\left(n_{1}\right)$ such that $y_{n} \geq c_{1} \eta_{\alpha}(n)$ for $n \in N\left(n_{2}\right)$. Hence

$$
\sum_{n=n_{2}}^{\infty} f\left(n, c_{1} \eta_{\alpha}(n+1)\right)<\infty
$$

which contradicts (12). If $\triangle y_{n}<0$ for $n \in N\left(n_{1}\right)$, then by Lemma 1, there is a constant $c_{2}>0$ such that $y_{n} \geq c_{2} \eta_{\alpha}(n)$ for $n \in N\left(n_{1}\right)$.

By the strong superlinearity of $f$, we have

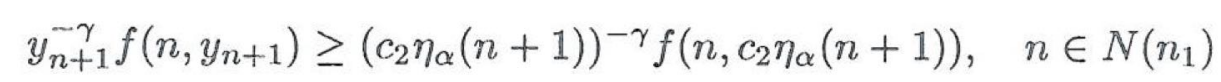


for some constant $\gamma>\alpha$. We see the difference

$$
\triangle\left\{-\left[-a_{n}\left(\triangle y_{n}\right)^{\alpha_{*}}\right]^{-(\gamma-\alpha) / \alpha}\right\}=\frac{\gamma-\alpha}{\alpha} t^{-\gamma / \alpha} f\left(n, y_{n+1}\right)
$$

where $-a_{n+1}\left(\triangle y_{n+1}\right)^{\alpha_{*}}<t<-a_{n}\left(\triangle y_{n}\right)^{\alpha_{*}}$. Using (13) and (5) in (14) we have

$$
\begin{aligned}
\Delta\left\{-\left[-a_{n}\left(\Delta y_{n}\right)^{\alpha_{*}}\right]^{-(\gamma-\alpha) / \alpha}\right\} \geq & \frac{\gamma-\alpha}{\alpha}\left[-a_{n+1}\left(\Delta y_{n+1}\right)^{\alpha_{*}}\right]^{-\gamma / \alpha} y_{n+1}^{\gamma} y_{n+1}^{-\gamma} f\left(n, y_{n+1}\right) \\
\geq & \frac{\gamma-\alpha}{\alpha}\left(-a_{n+1}\left(\triangle y_{n+1}\right)^{\alpha_{*}}\right)^{-\gamma / \alpha}\left(-a_{n+1}\left(\triangle y_{n}\right)^{\alpha_{*}}\right)^{\gamma / \alpha} X \\
& \eta_{\alpha}^{\gamma}(n+1)\left(c_{2} \eta_{\alpha}(n+1)\right)^{-\gamma} f\left(n, c_{2} \eta_{\alpha}(n+1)\right) \\
= & \frac{\gamma-\alpha}{\alpha} c_{2}^{-\gamma} f\left(n, c_{2} \eta_{\alpha}(n+1)\right), \quad n \in N\left(n_{1}\right) .
\end{aligned}
$$

Summation of the above yields

$$
\frac{\gamma-\alpha}{\alpha} c_{2}^{-\gamma} \sum_{s=n_{1}}^{n} f\left(s, c_{2} \eta(s+1)\right) \leq\left[-a_{n}\left(\triangle y_{n_{1}}\right)^{\alpha_{*}}\right]^{-(\gamma-\alpha) / \alpha}, \quad n \in N\left(n_{1}\right)
$$

which implies that $\sum_{n=n_{1}}^{\infty} f\left(n, c_{2} \eta_{\alpha}(n+1)\right)<\infty$, again contradicting (12). This completes the proof of the theorem.

Theorem 7. Let equation (1) be strongly sublinear. All solutions of equation (1) are oscillatory if and only if

$$
\sum_{n=n_{0}}^{\infty}\left(\frac{1}{a_{n}} \sum_{s=n_{0}}^{n-1}|f(s, c)|^{1 / \alpha}\right)=\infty
$$

for every nonzero constant $c$.

Proof. It is enough to prove sufficient part, since the necessary part follows from Theorem 2. Let condition (15) holds and equation (1) has a nonoscillatory solution $\left\{y_{n}\right\}$ which is positive for $n \in N\left(n_{0}\right)$.

If $\Delta y_{n}>0$ for $n \geq n_{1} \in N\left(n_{0}\right)$, then there is a constant $c_{1}>0$ such that $\sum_{n=n_{1}}^{\infty} f\left(s, c_{1}\right)<\infty$, and this together with the condition $\sum_{n=n_{1}}^{\infty} a_{n}^{-1 / \alpha}<\infty$ shows that

$$
\sum_{n=n_{1}}^{\infty}\left(\frac{1}{a_{n}} \sum_{s=n_{1}}^{n-1} f\left(s, c_{1}\right)\right)^{1 / \alpha} \leq \eta_{\alpha}\left(n_{1}\right)\left(\sum_{n=n_{1}}^{\infty} f\left(s, c_{1}\right)\right)<\infty
$$

which contradicts (15).

If $\Delta y_{n}<0$ for $n \in N\left(n_{1}\right)$, then summation of equation (1) gives $\sum_{s=n_{1}}^{n-1} f\left(s, y_{s+1}\right) \leq$ $a_{n}\left(-\Delta y_{n}\right)^{\alpha}, \quad n \in N\left(n_{1}\right)$ (cf. (7)), or equivalents

$$
-\triangle y_{n} \geq\left(\frac{1}{a_{n}} \sum_{s=n_{1}}^{n-1} f\left(s, y_{s+1}\right)\right)^{1 / \alpha}, \quad n \in N\left(n_{1}\right)
$$


Since $y_{n} \leq c_{2}$ for $n \in N\left(n_{1}\right)$, for some constants $c_{2}>0$, the strongly sublinearity implies

$$
y_{n+1}^{-\partial} f\left(n, y_{n+1}\right) \geq c_{2}^{-\partial} f\left(n, c_{2}\right), \quad n \in N\left(n_{1}\right)
$$

for some $\partial<\alpha$. Combining (16) with (17) and using the decreasing property of $\left\{y_{n}\right\}$, we see that

$$
\begin{aligned}
-\Delta y_{n} & \geq c_{2}^{-\partial / \alpha} a_{n}^{-1 / \alpha}\left(\sum_{s=n_{1}}^{n-1} y_{s+1}^{c} f\left(s, c_{2}\right)\right)^{1 / \alpha} \\
& \geq c_{2}^{-\partial / \alpha} a_{n}^{-1 / \alpha} y_{n+1}^{\partial / \alpha}\left(\sum_{s=n_{1}}^{n-1} f\left(s, c_{2}\right)\right)^{1 / \alpha}, \quad n \in N\left(n_{1}\right) .
\end{aligned}
$$

Now, we consider the difference $\triangle\left(-\left(y_{n}\right)^{\alpha-\partial / \alpha}\right)$;

$$
\triangle\left(-\left(y_{n}\right)^{\alpha-\partial / \alpha}\right)=-\frac{(\alpha-\partial)}{\alpha} t^{-\partial / \alpha} \triangle y_{n}
$$

where $y_{n+1}<t<y_{n}$ and $\left\{y_{n}\right\}$ is decreasing.

From (18) and (19) we have

$$
\begin{aligned}
\Delta\left(-\left(y_{n}\right)^{\alpha-\partial / \alpha}\right) & \geq \frac{\alpha-\partial}{\alpha} c_{2}^{-\partial / \alpha} a_{n}^{-1 / \alpha} y_{n+1}^{\partial / \alpha}\left(\sum_{s=n_{1}}^{n-1} f\left(s, c_{2}\right)\right)^{1 / \alpha} y_{n+1}^{-\partial / \alpha} \\
& =\frac{\alpha-\partial}{\alpha} c_{2}^{-\partial / \alpha} a_{n}^{-1 / \alpha}\left(\sum_{s=n_{1}}^{n-1} f\left(s, c_{2}\right)\right)^{1 / \alpha}, \quad n \in N\left(n_{1}\right) .
\end{aligned}
$$

Summation of last inequality yields

$$
\frac{\alpha-\partial}{\alpha} c_{2}^{-\partial / \alpha} \sum_{s=n_{1}}^{n-1}\left(\frac{1}{a_{s}} \sum_{i=n_{1}}^{s-1} f\left(i, c_{2}\right)\right)^{1 / \alpha} \leq\left(y_{n_{1}}\right)^{(\alpha-\partial) / \alpha}
$$

for $n \in N\left(n_{1}\right)$, which again contradicts (15). This complete the proof.

Example 2. Consider the difference equation

$$
\triangle\left(2^{\lambda n}\left(\triangle y_{n}\right)^{\alpha_{*}}\right)+2^{\mu n} y_{n+1}^{\beta_{*}}=0, . n \in \mathbb{N}
$$

which is the same as (11).

Let $\alpha<\beta$. Then, by Theorem 6 all solutions of (20) are oscillatory if and only if $\mu / \lambda \geq \beta / \alpha$. Let $\alpha>\beta$. Then, by Theorem 7 all solutions of (20) are oscillatory if and only if $\mu \geq \lambda$.

Remark. The results of this paper generalize some of the results of $\mathbb{H e}[4]$ and Zhang [17]. Also the results of this paper generalize some of the results obtained in [10] in the sense that here we do not require the condition $a_{n} \rightarrow \infty$ as $n \rightarrow \infty$. 


\section{References}

[1] R. P. Agarwal, Difference Equations and Inequalities, Marcel Dekker, New York, 1992.

[2] T. J. Bronmwich, An Introductin to the Theory of Infinite Series, Mac Millan, London, 1926.

[3] J. W. Hooker and W. T. Patula, "A second order nonlinear difference equation: Oscillation and asymptotic behavior," J. Math. Anal. Appl. 91 (1983), 9-29.

[4] X. Z. He, "Oscillatory and asymptotic behavior of second order nonlinear difference equations," J. Math. Anal. Appl. 175 (1993), 482-498.

[5] M. R. S. Kulenvic and M. Budincevic, "Asymptotic analysis of nonlinear second order difference equations," An. Stiint. Univ. Iasi Sect. I a mat (N. S) 23 (1984), 39-52.

[6] V. Lakshmikantham and D. Trigiante, Theory of Difference Equations: Numerical Methods and Applications, Academic Press, New York, 1988.

[7] J. Popenda, "The oscillation of solutions of difference equations," Comput. Math. Appl. 28 (1994), 271-279.

[8] B. Szmanda, "Characterization of oscillation of second order nonlinear difference equations," Bull. Polish. Acad. Sci. Math. 34 (1986), 133-141.

[9] E. Thandapani, "Oscillatory behavior of solutions of second order nonlinear difference equations," J. Math. Phy. Sci. 25 (1991), 457-464.

[10] E. Thandapani, G. R. Graef and P. W. Spikes, "On the oscillation of solutions of second order quasilinear difference equations," Nonlinear World 3 (1996), 545-565.

[11] E. Thandapani, M. Maria Susai Manuel and R. P. Agarwal, "Oscillation and nonoscillation theorems for second order quasilinear difference equations," Facta Universitatis Series: Mathematics and Informatics (to appear).

[12] E. Thandapani, and R. Arul, "Oscillation and nonoscillation theorems for a class of second order quasilinear difference equatios," (to appear).

[13] P. J. Y. Wong and R. P. Agarwal, "Oscillation and nonoscillations of halflinear difference equations generated by deviating arguments," Advances in difference Equations II Computers and Mathematics with Applications (to appear).

[14] P. J. Y. Wong and R. P. Agarwal, "Oscillation theorems and existence of positive monotone solutions for second order nonlinear equations," Math. Comp. Modelling 21 (1995), 63-84.

[15] P. J. Y. Wong and R. P. Agarwal, "Oscillations and monotone solutions of a second order quasilinear difference equations" (to appear).

[16] P. J. Y. Wong and R. P. Agarwal, "On the oscillation and asymptotically monotone solutions of second order quasilinear differential equations," Appl. Math. Comp. (to appear).

[17] B. G. Zhang, "Oscillation and asymptotic behavior of second order difference equtions," J. Math. Anal. Appl. 173 (1993), 58-68.

Department of Mathematics, Madras University P. G. Centre, Salem-636 011, TamilNadu, INDIA. 